\title{
Políticas públicas para a saúde do idoso: revisão sistemática
}

\author{
Public policies for the elderly's health: systematic review \\ Políticas públicas para la salud del anciano: revisión sistemática
}

\author{
Alessandra Conceição Leite Funchal Camacho', Maria José Coelho' \\ 'Universidade Federal do Rio de Janeiro. Escola de Enfermagem Anna Nery. Rio de Janeiro, RJ
}

Submissão: $31 / 10 / 2008$

Aprovação: $11 / 03 / 2010$

\section{RESUMO}

O objetivo deste trabalho é analisar o desenvolvimento dos programas de saúde do idoso com base nas referências contidas nas principais bases de dados. Estudo de revisão sistemática de natureza Quanti-Qualitativa no período de 01/09/08 à 30/09/08 nas bases de dados da Biblioteca Virtual da Saúde. Para análise das informações foi realizada a organização do conteúdo Quanto ao ano, tipo de publicação e métodos/técnicas, essência do conteúdo e produção do conhecimento, base de dados, recomendações dos autores. Os resultados apontam para o desenvolvimento de pesquisas voltadas para a divulgação e resultados das políticas de saúde e o seu conhecimento pelos profissionais de saúde que cuidam de idosos sendo necessário um direcionamento e capacitação específica.

Descritores: Políticas Públicas de Saúde; Idoso; Enfermagem.

\section{ABSTRACT}

The objective of this work was to analyze the development of health of the elderly based on references contained in the main databases. Study of systematic review considering the period from 01/09/08 to 30/09/08 in the databases of the Library of Health to check that the information was held to organize the content on the year, publication type and methods / technieues, content and essence of the production of knowledge, database, the authors' recommendations. The results emphasize the development of research focused on the dissemination and results of health policies and their knowledge by health professionals who care for elderly is a necessary direction and specific training.

Key words: Health Public Policy; Aged; Nursing.

\section{RESUMEN}

El objetivo es analizar el desarrollo de las políticas públicas de salud para los ancianos en las referencias Que figuran en las principales bases de datos. Estudio de revisión sistemática de naturaleza cuanti-cualitativa en el período comprendido entre 01/09/08 al 30/09/ 08 en la Biblioteca Virtual de Salud. Para análisis de las informaciónes se realizó una organización del contenido sobre el año, tipo de publicación y métodos/técnicas, contenido y la esencia de la producción de conocimiento, base de datos, recomendaciones de los autores. Los resultados apuntan para el desarrollo de investigaciones Que hacen la difusión sobre las políticas de salud y su conocimiento por los profesionales de salud que cuidan de ancianos pues es necesaria una dirección y una formación específica.

Descriptores: Políticas Públicas de salud; Ancianos; Enfermería. 


\section{INTRODUÇÃO}

O envelhecimento é um processo universal Que é compreendido por uma redução das atividades funcionais e possui algumas tendências em relação as enfermidades Que levam continuamente a construção de políticas públicas para o idoso tanto no âmbito internacional assim como principalmente no âmbito brasileiro. Essas políticas estão voltadas não somente para a terceira idade como também para os profissionais da saúde visando a sua divulgação e implementação. Desta forma, é verificado um aumento no contingente populacional dos idosos em virtude da baixa de natalidade, aumento da expectativa de vida, desenvolvimento de novas tecnologias Que vislumbraram tratamentos Que até alguns anos atrás eram impensados uma perspectiva e um prognóstico de vida favorável para algumas enfermidades.

Verifica-se em projeções palpáveis Que no último censo 2000 do Instituto Brasileiro de Geografia e Estatística a população de 60 anos ou mais de idade no Brasil era de 14.536.029 de pessoas, contra 10.722 .705 em 1991. O peso relativo da população idosa no início da década de noventa representava 7,3\%, enQuanto em 2000 essa proporção atingia $8,6 \%$. Considerando a continuidade das tendências verificadas para as taxas de fecundidade e longevidade da população brasileira as estimativas para os próximos 20 anos indicam Que a população idosa poderá exceder 30 milhões de pessoas ao final deste período, chegando a representar $13 \%$ da população no país ${ }^{(1)}$.

Em meio a essa transição demográfica, é importante ressaltar Que, juntamente com a modificação observada e descrita sob o aspecto Quantitativo, precisamos estar atentos aos aspectos Que ultrapassam a Quantidade, já Que nos preocuparmos com a Qualidade de vida dessa população. Essa mudança no perfil epidemiológico do país é resultante de transformações da vida da população nos âmbitos social, econômico e cultural acarretando novos olhares e planejamentos, para contemplarem suas expectativas de saúde, bem como atender às demandas biopsicossociais visando ao equilíbrio do processo vital ${ }^{(2)}$.

Assim, temos como objetivo neste artigo analisar o desenvolvimento dos programas de saúde do idoso na área da saúde com base nas referências contidas nas principais bases de dados da Biblioteca Virtual da Saúde (BVS). Podemos identificar as seguintes políticas públicas de saúde Que precisam constantemente de reconhecimento e são objeto de nossa atenção, para o cuidado ao idoso: Política Nacional do Idoso - Lei 8.842 de 1994; Portaria 702 de 2002 Que cria mecanismos de organização e implantação de Redes Estaduais de Assistência à Saúde do Idoso tendo como base as condições de gestão e a divisão de responsabilidades definida pela Norma Operacional de Assistência à Saúde (NOAS); Portaria 703 de 2002 Que institui no âmbito do SUS o Programa de assistência aos Portadores de Doença de Alzheimer; Protocolo de Tratamento da Doença de Alzheimer - Portaria n. ${ }^{\circ} 843$ de 2002; Estatuto do Idoso - Lei 10.741 de 2003 e, a Política Nacional de Saúde da Pessoa Idosa - Portaria n. ${ }^{\circ} 2.528$ de 2006.

Com propriedade percebemos Que as políticas públicas em saúde devem se apoiar em informações objetivas respaldadas por evidências científicas. A Saúde Pública e a Epidemiologia têm um importante papel nesse processo, seja por meio do desenvolvimento de pesquisas, como pela coleta sistemática de informações oriundas dos sistemas de vigilância Que possibilita a avaliação sistemática de dados sobre magnitude, escopo, características e conseqüências das doenças ${ }^{(3)}$.

Verificando o desenvolvimento e as mudanças nas políticas públicas para amparar de forma adequada o idoso, há necessidade de uma reorientação dos serviços de saúde, investindo-se principalmente na atenção básica com rediscussões de estratégias preventivas e de promoção à saúde ${ }^{(2)}$. Por outro lado, os profissionais de saúde, principalmente aQueles que atuam na rede de atenção básica devem ser alvo de treinamento e capacitação continuados para se adequarem às necessidades da população idosa. Levando em consideração Que o ambiente familiar constituise uma principal fonte de apoio ao idoso, há que se estimular o fortalecimento das relações familiares com o propósito de se minimizarem as dificuldades e angústias vivenciadas por ambos, idosos e familiares ${ }^{(4-5)}$.

Portanto, Quanto maior for o acesso aos bens e serviços da sociedade, maior será a Qualidade de vida no processo de envelhecimento. E sob esta ótica, os serviços de saúde têm papel fundamental na atenção à saúde, para Que a população idosa possa usufruir a vida com tudo aquilo Que construiu. Para tal, são reQueridos investimentos Que priorizem a prevenção de doenças e controle de condições de cronicidade Que permita aos idosos um viver com Qualidade $\mathrm{e}^{(5)}$.

A relevância deste artigo está no fato de compreender Que assistência de saúde ao idoso exige dos serviços de saúde e, também da equipe de saúde uma Qualificação diferenciada, porQue são estes profissionais Que estão na "linha de frente" do cuidado a esta clientela. A contribuição deste artigo visa também à possibilidade de reduzir assim a incidência de complicações da saúde do idoso com o conhecimento de políticas públicas de saúde no Brasil visando uma articulação das ações de saúde entre os profissionais de saúde.

\section{METODOLOGIA}

Para viabilizar o desenvolvimento deste estudo, optamos por um estudo de revisão sistemática de natureza Quanti-Qualitativa. O período de coleta de dados se desenvolveu de 01/09/08 à 30/ 09/08 nas seguintes bases de dados da Biblioteca virtual da Saúde: Lilacs (Literatura Latino Americana e do Caribe em Ciências da Saúde), SciELO (Scientific Eletronic Library Online) e Bdenf (Base de Dados da Enfermagem).

A revisão sistemática é o delineamento de um estudo secundário através de outros estudos, ditos primários, Que são analisados de forma criteriosa e avaliados Quanto à sua Qualidade científica para serem incluídos, ou não, numa análise estatística, a metanálise. Um outro aspecto relevante é Que as revisões sistemáticas devem ser desenvolvidas antes de QualQuer projeto de pesquisa clínica ${ }^{(6)}$. Foram utilizados os seguintes descritores: Políticas Públicas de Saúde; Idoso; Enfermagem.

Os Critérios de Inclusão das referências foram os seguintes: Possuir aderência ao objetivo proposto; conter articulação com os Programas de Saúde referidos. A análise das referências foi baseada nas publicações dos últimos cinco anos (de 2003 a 2008) em virtude da grande maioria dos programas estarem contidos 
neste período.

Realizamos uma leitura flutuante inicial das referências e, portanto, das 67 referências encontradas na Lilacs somente 24 referências tiveram aderência ao nosso objetivo; das 18 referências encontradas na SciELO 14 referências tiveram aderência e das seis referências na BDENF Quatro referências foram aproveitadas dentro dos critérios de inclusão estabelecidos.

$\mathrm{Na}$ base dados da Scielo das 14 referências selecionadas: duas eram da área de Nutrição; duas da área de Odontologia e 10 referentes a todas as áreas de conhecimento da saúde e da enfermagem. Portanto, ao realizar uma leitura em profundidade selecionamos 10 referências relacionadas que de fato tratavam de políticas públicas de saúde ao idoso. $\mathrm{Na}$ base de dados Bdenf das Quatro referências encontradas somente uma foi selecionada porQue duas referências se tratavam das políticas públicas da Espanha e Que não se adequou a nossa realidade e, uma referência também foi encontrada na Scielo. Na base de dados Lilacs das 24 referências selecionadas foram aproveitadas 15 porque duas referências se repetiram na Bdenf e sete referências se repetiram na SciELO. Portanto, as referências Que foram analisadas são 26 sendo 10 na base de dados Scielo, uma na BDENF e 15 na LILACS.

\section{RESULTADOS E DISCUSSÃO}

Para análise das informações foi realizada a organização do conteúdo encontrado Quanto ao ano, tipo de publicação e métodos/ técnicas utilizadas, essência do conteúdo e produção do conhecimento, base de dados, recomendações dos autores.

Portanto, o Quantitativo de referências por ano encontramos cerca de 23\% das referências no ano de 2008; $31 \%$ das referências no ano de 2007; 23\% das referências no ano de 2006; $8 \%$ de referências no ano de 2005 e 15\% de referências no ano de 2004 . Este demonstrativo já nos leva a afirmar Que no período de 2004 a 2008 ocorreu um aumento de publicações Que tratam de profissionais na área da saúde operacionalizando as políticas públicas de saúde voltada para o idoso.

Sobre o Quantitativo de referências encontradas nas bases de dados deste estudo encontramos $19 \%$ das referências contidas na Scielo, $61 \%$ das referências estiveram contidas na base Lilacs e $4 \%$ das referências foram encontradas na Bdenf. Ocorreram 12\% das referências estiveram contidas tanto nas bases da Lilacs como da Scielo e 4\% das referências foram encontradas nas bases LILACS, SCiELO e BDENF.

Ao analisar o tipo de publicação no Que tange o seu método e técnicas de pesquisa utilizadas encontramos cerca de 36\% das referências trazem a pesQuisa de campo; 13\% das referências trabalham em seu conteúdo a revisão de literatura; $21 \%$ desenvolveram a entrevista; $4 \%$ das referências realizaram a observação; 13\% das referências aplicaram um Questionário estruturado; 11\% das referências desenvolveram análise documental e $2 \%$ das referências realizaram o método de estudo de caso. Neste período as publicações estiveram voltadas em sua maior parte para a pesquisa de campo em detrimento das demais modalidades, vislumbrando a necessidade de conhecer a aplicabilidade e grau de resolutividade das políticas públicas de saúde ao idoso.

Sobre a essência do conteúdo estudado nas referências e as suas produções no conhecimento encontramos $8 \%$ referências Que apontam as desigualdades no acesso e uso dos serviços de saúde por idosos; 22\% referências analisam os dados demográficos, sócio-econômicos, padrão epidemiológico e as condições de saúde dos idosos em relação as dificuldades no acesso aos serviços de saúde; $4 \%$ das referências pautaram suas discussões na construção de um sistema integrado de informações geográficas e de condições de saúde para idosos com demência, cadastrados nas Unidades de Saúde da Família de um município paulista e 4\% das referências demonstraram as fontes de dados secundários dos sistemas de informação como relevantes para estudos das políticas de saúde direcionadas aos idosos.

Com relação as Questões econômicas e sociais sofrida pelo idoso, há ainda Questões básicas como solidariedade e dificuldade de inserção social; entende-se Que a família, por sua posição nuclear, é responsável por cuidar da pessoa idosa. Por outro lado, o abrigamento asilar só deve ocorrer no caso de completa ausência de parentes ou como medida preventiva para garantir a sobrevivência da pessoa idosa. Na prática a realidade é outra: situações de humilhação, insultos e maus tratos são a cada dia mais comum no cotidiano das populações de pessoas idosas; o determinante é a escassez de entendimento por parte da sociedade, predominantemente composta por jovens, em relação ao envelhecimento e suas peculiaridades ${ }^{(7)}$.

Encontramos 8\% das referências Que tratam sobre o envelhecimento da população brasileira e a mudança na sua estrutura etária requerendo das políticas públicas e dos profissionais de saúde atitudes definidas na abordagem de atenção à saúde com ênfase no trabalho interdisciplinar com vistas a preservar a autonomia, a participação, o cuidado, a auto-satisfação e a possibilidade de o idoso atuar em variados contextos sociais. Também encontramos 8\% das referências Que tratam de programas de atividade física para idosos em diferentes contextos e a educação postural.

Percebemos Que Quando o convívio social não é suficiente para direcionar os indivíduos a respeitarem o modo de viver, com princípios éticos e morais, de determinado grupo, é necessário Que os princípios se transformem em leis através de políticas públicas de saúde. Transformar as leis em políticas de governo é o compromisso de oferecer estratégias para fazer com Que as regras estabelecidas sejam respeitadas por todos ${ }^{(8)}$. Ainda sobre as referências Que destacam sobre atividade física esta é um meio de modificar os hábitos de vida dos idosos, retardando o aparecimento de doenças crônicas e mantendo-os por mais tempo funcionalmente capazes para realizar as tarefas da vida diária ${ }^{(9)}$.

Apenas 4\% das referências discutem o acesso dos idosos as medicações para o seu tratamento de saúde afirmando Que grande maioria adQuirem as medicações através de seus próprios rendimentos; 18\% das referências tratam sobre os familiares cuidadores destacando o papel do cuidador informal no cuidado do idoso dependente (com AVC, Doença de Alzheimer e outras Síndromes Demenciais) além da a vivência de ser um cuidador familiar e os modelos de atenção e suporte direcionados ao cuidador informal. Constatou-se, ainda nestas referências, Que a família desempenha papel importante na reabilitação dos idosos acometidos e Que determina mudanças no estilo de vida.

A necessidade de cuidados ininterruptos, o difícil manejo das 
manifestações psiQuiátricas e comportamentais, somadas às vivências dos laços emocionais, tanto positivos como negativos experienciados pelo convívio anterior à instalação da doença, produzem desgaste físico, mental e emocional. Assim, os efeitos psicossociais da doença fazem do cuidador uma importante entidade no contexto das investigações científicas e da conseQüente sobrecarga, experimentado pelo cuidador ao realizar uma gama de atividades potencialmente geradoras de estresse e efeitos negativos $^{(10)}$. Além disso, ante as situações de doenças, sobretudo Quando se verifica comprometimento da saúde, as pessoas devem se submeter a formas de adaptação. Nesse novo contexto, o idoso necessita de uma série de medidas planejadas e desenvolvidas com vistas a lhe facultar a maior integralidade possível. Como procedimento essencial nesse processo sobressai a realização de ações para a reabilitação dos déficits neurológicos surgidos ${ }^{(1)}$.

No entanto, a preocupação com as medicações citadas nas referências estão baseadas nos extensos gastos públicos bem como os gastos dos próprios idosos; custo específico de uma medicação em detrimento de outra; prescrições excessivas Que poderiam ser reavaliadas e o uso excessivo de medicamentos que poderiam ser evitados $^{(12)}$.

Temos 4\% das referências Que tratam sobre os possíveis sinais de violência observados pelos entrevistadores nos idosos; $4 \%$ das referências avaliam as políticas públicas de atenção a pessoa idosa, bem como sua evolução, tendo como ambiência o Estatuto do Idoso; 8\% das referências analisam Quem as políticas indicam possibilidades de trabalhos comunitários e institucionais em Que o idoso tenha papel de relevância na sociedade a partir de suas experiências passadas e presentes, para contribuir com a conscientização de outras gerações sobre Questões ambientais e a nível ambulatorial em Manaus (Programa de Atenção à Saúde do Idoso - PASI).

Percebemos Que embora leis existam, ainda há muito Que se elaborar no "iceberg" de violência contra o idoso, porQue, ainda Que tenha leis de amparo ao idoso há o viés dos agressores Que muitas vezes são seus próprios familiares Que deveriam na realidade ampará-lo e não o fazem. Para o idoso pode ser angustiante utilizar estas leis para denunciar estes agressores, seus familiares ${ }^{(13)}$.

Porém, tivemos $8 \%$ das referências que tratam do tratamento da Síndrome da Imunodeficiência Adeuirida (SIDA) verificando seus possíveis significados aos idosos sobre a concepção da velhice e envelhecimento de acordo com as culturas e contextos históricos diferenciados e a Tuberculose em idosos com menos sintomas e menor número de abandonos durante seu período de tratamento.

Sobre a recomendação dos autores encontramos 19,7\% das referências Que observaram desigualdades no uso e acesso aos serviços de saúde e inadeQuação do modelo de atenção, indicando necessidade de políticas públicas Que levem em conta as especificidades dessa população, facilitem o acesso e possam reduzir essas desigualdades; $7,7 \%$ das referências indicam Que seus estudos contribuem para dar maior visibilidade a essa parcela populacional, para compreensão do processo de envelhecimento e adequação das políticas públicas e Que são necessários os princípios norteadores da gestão do Sistema Único de Saúde e, também, uma articulação impressiva por parte do poder público em torno do tema.

A desigualdade no uso e no acesso aos serviços de saúde temos a associação de renda, escolaridade, sexo e morbidade referida traduzem a grande necessidade e a baixa capacidade de utilização. A relação dos idosos com os serviços de saúde são intensas podendo traduzir injustiças e inadeQuações que impactam na Qualidade de vida dessa população e Que, por sua vez, depende de políticas públicas integradas e efetivas ${ }^{(14)}$.

Verificamos 3,8\% das referências Que tratam da utilização de tecnologias de geoprocessamento. Estas se mostraram importantes no planejamento de ações de cuidado em gerontologia, contribuindo para a gestão de programas públicos de saúde; 3,8\% das referências propõem um roteiro para a realização do InQuérito Nacional de Saúde; 7,7\% das referências indicam Que houve redução, estatisticamente significativa, das taxas de prevalência de incapacidade funcional, em mobilidade física, entre as pessoas idosas; 3,8\% das referências destacam a tendência das políticas públicas em saúde Que preconizam a humanização da atenção, a promoção da saúde, educação e desenvolvimento da ciência, tecnologia e inovação em saúde, dentre outras, a tônica no discurso e na prática requer a efetiva elaboração e operacionalização interdisciplinar.

Uma informação relevante é Que a utilização de tecnologias de geoprocessamento nos serviços públicos de saúde é de extrema importância, pois permite uma análise da combinação de fatores de risco em um determinado local, Que podem levar à maior fragilidade dos idosos. Estas análises certamente trarão contribuições significativas para o cuidado ao idoso com demência ${ }^{(15)}$. No entanto, as equipes multiprofissionais e multidisciplinares formam etapas para a interação e para a interdisciplinaridade. Nelas ocorrem a justaposição e a integração de métodos, teorias e conhecimentos, e a decisão é sempre tomada pelo chefe da equipe. Na interdisciplinaridade, a decisão é compartilhada pelos membros da equipe e a liderança é rotativa ${ }^{(16)}$.

Ocorreram $7,7 \%$ das referências Que indicam uma proposta diretiva de programas e ações no âmbito das políticas públicas para o município de Florianópolis, na forma de rede articulada entre os diferentes setores da administração pública, para o desenvolvimento das atividades físicas coordenadas com vistas à promoção do envelhecimento saudável. Ainda nestas referências os autores indicam a necessidade de maiores investimentos na área de saúde e treinamento dos recursos humanos que trabalham com os idosos nos serviços de saúde para facilitar o acesso ao atendimento adequado de saúde dos idosos no município de Florianópolis.

O envelhecimento é, hoje, uma realidade Que não pode ser ignorada na maioria das sociedades desenvolvidas e em desenvolvimento, tornando-se temática relevante do ponto de vista científico e de políticas públicas, mobilizando pesquisadores e promotores de políticas sociais, na discussão do desafio Que a longevidade humana está colocando para as sociedades ${ }^{(17)}$.

Encontramos 3,8\% das referências Que indicam os riscos identificados na polifarmárcia e mostram uma necessidade de políticas públicas Que visem promover o uso racional de medicamentos; $3,8 \%$ das referências apontam Que os profissionais de saúde devem lutar pela implantação de políticas públicas Que facilitem a reabilitação, e os enfermeiros devem centrar sua atuação na educação para a saúde e na assistência sistematizada, privilegiando os cuidados domiciliares essenciais para a reabilitação. 
Apenas 3,8\% das referências tratam Que a meta é contribuir para a melhora do nosso sistema de saúde como um todo, mas, particularmente, para o segmento dos idosos, grupo etário Que mais cresce no país e Que necessita de políticas afirmativas e integradoras Que priorizem uma abordagem preventiva, com programas abrangentes de educação, sem abandonar uma assistência de Qualidade para aqueles idosos com doenças adeuiridas; $7,7 \%$ dos estudos demonstram a importância da ampliação de estratégias Que tenham o cuidador como sujeito principal, cabendo ao profissional de saúde e às políticas públicas valorizem a rede de suporte ao idoso dependente. Esta rede é importante como base do processo de cuidar com Qualidade. Estas duas últimas referências destacam Que a sobrecarga produzida pelas demandas de cuidados pode ser minimizada, pela adoção de estratégias e de políticas públicas eficazes, representando melhor Qualidade de vida para o idoso e seu cuidador.

Estes fatores identificados devem-se ao processo acelerado e diferenciado de envelhecimento no Brasil em detrimento dos países mais desenvolvidos. Há padrões semelhantes no Que diz respeito, por exemplo, aos diferenciais sociodemográficos na prevalência de incapacidade funcional Que são específicos conforme mostram algumas referências ${ }^{(18)}$. Sobre o cuidador do idoso como sujeito importante para a seqüência do cuidado no domicílio existe a necessidade de programas voltados para estes para que a atividade do cuidado seja efetiva e investida através de práticas adeQuadas, trazendo benefícios para Quem cuida e Quem recebe este cuidado ${ }^{(19)}$.

Há 3,8\% das referências Que apontam uma projeção significativa de idosos Que sofreram violência doméstica dando a importância da formulação de políticas públicas Que visem a proteger esta faixa da população; 3,8\% das referências tratam da importância de trabalharem as políticas públicas tornando-as mais clara e rápida a execução de práticas primordiais para a melhora da Qualidade de vida individual, da Qualidade do ambiente onde vivem e da Qualidade das relações interpessoais.

Em geral temos uma população idosa heterogênea em diversos aspectos sociais, econômicos, de gênero, culturais, psicológicos dentre outros. Diante disso, observa-se a importância do planejamento de políticas públicas direcionadas às necessidades específicas da população idosa, além de outros estudos Que possibilitem ampliar o conhecimento a respeito dessa população ${ }^{(20)}$.

Ocorreram $7,7 \%$ das referências Que indicam as dificuldades do diagnóstico, demora no início do tratamento e doenças associadas, têm maiores riscos de letalidade e de complicações durante o tratamento, principalmente entre os mais velhos. Preconizam uma atuação mais atenta e capacitada dos profissionais, bem como políticas públicas Que contemplem este segmento etário necessitando de maior adequação dos profissionais e serviços de saúde para uma efetiva implementação de políticas públicas de atenção adeQuada aos idosos.

Apenas 3,8\% das referências indicam Que a literatura examinada contribui para a formação do acadêmico de enfermagem e possibilita o intercâmbio de conhecimento entre profissionais da saúde sobre o tema idoso; $3,8 \%$ das referências evidenciam a importância de nos prepararmos Qualitativa e Quantitativamente para os vindouros perfis demográfico e epidemiológico da população, assim como rever ações e serviços atualmente ofertados aos idosos; 3,8\% das referências indicam a necessidade de criação de políticas públicas voltadas para a promoção de saúde do idoso e campanhas de prevenção a Doenças Sexualmente Transmissíveis/ SIDA direcionadas a esse segmento, proporcionando assim, Qualidade de vida para os idosos.

\section{CONSIDERAÇÕES FINAIS}

De fato não temos como ignorar Que o nosso desafio maior do século XXI será cuidar de uma população de mais de 32 milhões de idosos, a maioria com níveis socioeconômico e educacional baixos e uma alta prevalência de doenças crônicas não transmissíveis. O processo de envelhecimento tem um impacto significativo em inúmeros fatores Que afetam o desenvolvimento das sociedades e o relativo bem-estar, não só das pessoas idosas, mas também das populações jovens. Por se tratar de um grupo etário em rápido crescimento no mundo, as características sociodemográficas são imprescindíveis para o estabelecimento, viabilidade e integração de políticas públicas de saúde Que visem promover a saúde da população e controlar as doenças.

Percebemos que as políticas públicas de saúde destacam Que a família é a primeira fonte de suporte a Qual seus membros recorrem para resolução de problemas. Ela pode estar presente desde a simples preparação da refeição, passando pela modalidade de lazer, até a rotina do uso de medicamentos e de consultas para avaliação do estado de saúde.

Esta realidade demográfica e epidemiológica brasileira aponta para a urgência de mudanças e inovação nos modelos de atenção à saúde da população idosa e reclama estruturas criativas, com propostas de ações diferenciadas afim de Que o sistema ganhe efetividade e o idoso possa usufruir integralmente os anos proporcionados pelo avanço da ciência. Autonomia, participação, cuidado, auto-satisfação, possibilidade de atuar em variados contextos sociais e elaboração de novos significados para a vida na idade avançada são, hoje, conceitos-chave para QualQuer política destinada aos idosos.

Desta forma, concordamos Que em nossa sociedade, tanto do ponto de vista da organização social, como do legal, recai sobre a família a responsabilidade pelo cuidado para com as pessoas idosas, sem ser dado à mesma um preparo para tal função.

Verifica-se que as políticas de saúde para o idoso no cuidado familiar se transcrevem em relações de convivência no seio familiar e em sua relação com as instituições sócio-sanitárias podendo ser um meio de reprodução positiva destes programas de saúde do idoso. Para isso temos Que usar em nosso favor os avanços tecnológicos e o crescimento da renda das populações estão requerendo uma força de trabalho mais especializada, mesmo com o aumento da necessidade de cuidados da atenção básica.

As propostas destas portarias e lei fidedignas apontam para a divulgação de suas fontes para a população, bem como aos profissionais da área da saúde como um todo. Há a necessidade da realização de pesQuisas contínuas voltadas para a divulgação e o conhecimento dos profissionais de saúde que cuidam de idosos. É fundamental esta recomendação, pois a cada ano há um elevado número de atendimentos de idosos sendo necessária um direcionamento e capacitação específica para os profissionais de saúde. 


\section{REFERÊNCIAS}

I. Instituto Brasileiro de Geografia e Estatística. Perfil dos idosos responsáveis pelos domicílios no Brasil de 2000. Rio de Janeiro: IBGE; 2002

2. Barreira KS, Vieira LJES. O olhar da enfermagem para o idoso: revisão de literatura. Rev. enferm. UERJ 2004; I2(3): 0 I - 10.

3. Malta DC, Leal MC, Costa MFL, Neto OLM. Inquéritos Nacionais de Saúde: experiência acumulada e proposta para o ineuérito de saúde brasileiro. Rev Bras Epidemiol 2008; I I (1): 159-67.

4. Feliciano AB, Moraes SA, Freitas ICM. O perfil do idoso de baixa renda no Município de São Carlos, São Paulo, Brasil: um estudo epidemiológico. Cad Saúde Pública 2004; 20(6): 1575 -85.

5. Benedetti TB, Petroski EL, Gonçalves LT. Condições de saúde nos idosos de Florianópolis. Are Catarinenses Med 2006; 35(1): 44-5 I.

6. Bork AMT. Enfermagem Baseada em evidências. Rio de Janeiro: Guanabara Koogan; 2005.

7. Camargos CN, Mendonça CA, Viana EMB. Política, estado e sociedade: o estatuto do idoso e a atenção à saúde. Comun Ciênc Saúde 2006; 17(3):217-27.

8. Machado RFO, Velasco FCG, Amim V. O Encontro da Política Nacional da Educação Ambiental com a Política Nacional do Idoso. Saúde Sociedade 2006; 15(3): I62-9.

9. 9 Benedetti TRB, Gonçalves LHT, Mota JAPS. Uma proposta de política pública de atividade física para idosos. Texto Contexto Enferm 2007; 16(3): 387-98

10. Luzardo AR, Gorini MIPC, Silva APSS. Características de idosos com doença de Alzheimer e seus Cuidadores: uma série de casos em um serviço de Neurogeriatria. Texto Contexto Enferm 2006; 15(4): 587-94.
I1. Caetano IA, Damasceno MMC, Soares E, Fialho AVM. A vivência do processo de reabilitação após acidente vascular cerebral: um estudo Qualitativo. Online Brazilian Journal of Nursing [periódico da internet] 2007 Set [acesso em 2007 Set 09]; 6(2): [aprox 8 p.]. Disponível em: www.uff.br/nepae/ objn207caetanoetal.htm.

12. Carvalho MFC. A Polifarmácia em Idosos no Município de São Paulo - Estudo SABE - Saúde, Bem-estar e envelhecimento [dissertação]. São Paulo: Universidade de São Paulo; 2007.

13. Sanches APRA. Violência Doméstica contra idosos no município de São Paulo: estudo SABE [dissertação]. São Paulo: Universidade de São Paulo; 2006

14. Louvison MCP, Lebrão ML, Duarte YAO, Santos JLF, Malik AM, Almeida ES. Desigualdades no uso e acesso aos serviços de saúde entre idosos do município de São Paulo. Rev Saúde Pública 2008; 42(4): 733-40.

15. Pavarini SCL, Mendiondo EM, Montaño M, Almeida DMF, Mendiondo MSZ, Barham EJ, et al . Sistema de informações geográficas para a gestão de Programas municipais de cuidado a idosos. Texto Contexto Enferm 2008; 17(1): 17-25.

16. Saintrain MVL, Vieira LJES. Saúde bucal do idoso: abordagem interdisciplinar. Ciência \& Saúde Coletiva 2008; 13(4): 1 127-32.

17. Morais EP, Rodrigues RAPR, Gerhardt TE. Os idosos mais velhos no meio rural: realidade de vida e Saúde de uma população do interior gaúcho. Texto Contexto Enferm 2008; 17(2): 374-83.

18. Parahyba MI, Veras R. Diferenciais sociodemográficos no declínio funcional em mobilidade física entre os idosos no Brasil. Ciência \& Saúde Coletiva 2008; I3(4): 1257-64.

19. Moreira MD, Caldas CP. A Importância do Cuidador no Contexto da Saúde do Idoso. Esc Anna Nery R Enferm 2007; I I(3): 520-5. 